\title{
Intrinsic and chemo-sensitizing activity of SMAC- mimetics on high-risk childhood acute lymphoblastic leukemia
}

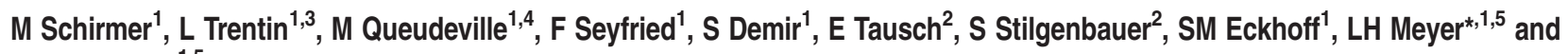 \\ K-M Debatin ${ }^{*, 1,5}$
}

SMAC-mimetics represent a targeted therapy approach to overcome apoptosis resistance in many tumors. Here, we investigated the efficacy of the SMAC-mimetic BV6 in B-cell precursor acute lymphoblastic leukemia (BCP-ALL). In ALL cell lines, intrinsic apoptosis sensitivity was associated with rapid cIAP degradation, NF- $\kappa$ B activation, TNF- $\alpha$ secretion and induction of an autocrine TNF- $\alpha$-dependent cell death loop. This pattern of responsiveness was also observed upon ex vivo analysis of 40 primograft BCPALL samples. Treatment with BV6 induced cell death in the majority of ALL primografts including leukemias with high-risk and poor-prognosis features. Inhibition of cell death by the TNF receptor fusion protein etanercept demonstrated that BV6 activity is dependent on TNF- $\alpha$. In a preclinical NOD/SCID/huALL model of high-risk ALL, marked anti-leukemia effectivity and significantly prolonged survival were observed upon BV6 treatment. Interestingly, also in vivo, intrinsic SMAC-mimetic activity was mediated by TNF- $\alpha$. Importantly, BV6 increased the effectivity of conventional induction therapy including vincristine, dexamethasone and asparaginase leading to prolonged remission induction. These data suggest SMAC-mimetics as an important addendum to efficient therapy of pediatric BCP-ALL.

Cell Death and Disease (2016) 7, e2052; doi:10.1038/cddis.2015.382; published online 14 January 2016

In childhood acute lymphoblastic leukemia (ALL), major improvements in therapy and supportive care have led to increased survival rates. ${ }^{1}$ In current treatment protocols, patients are assigned to multi-agent chemotherapy regimens of different intensities based on the patient's individual risk profile and probability to encounter relapse. ${ }^{2}$ However, $20 \%$ of patients relapse or do not respond well to therapy. ${ }^{3,4}$ Therefore, new treatment strategies are required to overcome resistance mechanisms and enhance outcome. Defects in cell death mechanisms contribute to resistance and treatment failure in many cancers, including leukemia. ${ }^{5,6}$ We previously described, that deficient apoptosis signaling in primary, patient-derived leukemia cells is indicative for early patient relapse and associated with rapid engraftment in NOD/SCID mice. ${ }^{7-10}$
Two major apoptosis signaling pathways are known, the death receptor (extrinsic) and mitochondrial (intrinsic) pathway. ${ }^{11}$ Within the extrinsic pathway, activation of death receptors of the tumor necrosis factor (TNF) superfamily activate caspase-8 via a death-inducing signaling complex (DISC) leading to activation of effector caspases. ${ }^{12}$ Activation of intrinsic signaling triggers mitochondrial release of cytochrome $c$ and SMAC (second mitochondria-derived activator of caspase). Released cytochrome $c$ forms a complex together with caspase-9, the apoptosome, which cleaves and activates caspase-3. SMAC antagonizes inhibitor of apoptosis proteins (IAPs), thus enabling apoptosis signaling. ${ }^{13}$

IAPs constitute a family of structurally related proteins, defined by the presence of at least one baculoviral IAP repeat (BIR) domain, which can bind to active subunits of caspases,

\footnotetext{
${ }^{1}$ Department of Pediatrics and Adolescent Medicine, Ulm University Medical Center, Ulm, Germany and ${ }^{2}$ Department of Internal Medicine III, UIm University Medical Center, Ulm, Germany

${ }^{*}$ Corresponding author: LH Meyer or K-M Debatin, Department of Pediatrics and Adolescent Medicine, Ulm University Medical Center, Eythstrasse 24, Ulm 89075, Germany. Tel: +4973150057254; Fax: +4973150057002; E-mail: lueder-hinrich.meyer@uniklinik-ulm.de or klaus-michael.debatin@uniklinik-ulm.de

${ }^{3}$ Present address: Department of Woman and Child Health, University of Padova, Padova, Italy.

${ }^{4}$ Present address: Department of Pediatric Hematology and Oncology, University Children's Hospital, Eberhard Karls University, Tuebingen, Germany.

${ }^{5}$ These authors contributed equally to this work.

Abbreviations: ALL, acute lymphoblastic leukemia; AML, acute myeloid leukemia; BCP-ALL, B-cell precursor acute lymphoblastic leukemia; BFM, Berlin Frankfurt Münster; BIR, baculoviral IAP repeat; cIAP, cellular inhibitor of apoptosis protein; CLL, chronic lymphoblastic leukemia; DISC, death-inducing signaling complex; DMSO, dimethyl sulfoxide; DSMZ, Deutsche Sammlung von Mikroorganismen und Zellkulturen; ETV6/RUNX1, Ets variant 6/Runt-related transcription factor; FADD, Fasassociated protein with death domain; HEPES, 4-(2-hydroxyethyl)-1-piperazineethanesulfonic acid; huCD19, human cluster of differentiation 19; I B, inhibitor of kappa B; IAP, inhibitor of apoptosis protein; $I C_{50}$, half-maximal inhibitory concentration; i.p., intraperitoneal; MLL/AF4, mixed lineage leukemia/ALL-1 fused gene on chromosome 4; MMP, mitochondrial membrane potential; NF-kB, nuclear factor kappa B; NIK, nuclear factor kappa B-inducing kinase; NOD/SCID, non-obese diabetic/severe combined immunodeficiency; PARP, poly(ADP-ribose)-polymerase; PB, peripheral blood; PBL, peripheral blood lymphocytes; RING, really interesting new gene; RIP1, receptorinteracting serine/threonine-protein kinase 1; RNA, ribonucleic acid; SMAC, second mitochondria-derived activator of caspase; shRNA, short hairpin RNA; siRNA, small interfering RNA; T-ALL, T-cell acute lymphoblastic leukemia; TNF, tumor necrosis factor; TNF- $\alpha$, tumor necrosis factor alpha; TNFR, tumor necrosis factor receptor; TP53, tumor protein p53; TTL, time to leukemia; TTR, time to leukemia reoccurrence; VDA, vincristine, dexamethasone, asparaginase; zVAD.fmk, N-benzyloxycarbonyl-Val-AlaAsp fluoromethylketone

Received 22.7.15; revised 24.11.15; accepted 26.11.15; Edited by H-U Simon.
} 
thereby inhibiting their function. Some IAPs (e.g., cIAP) also possess a RING domain conferring E3 ligase activity mediating ubiquitination reactions. ${ }^{14}$

Since IAPs are often overexpressed and associated with inferior outcome in different malignancies including childhood acute leukemias, they may serve as an attractive target for therapeutic intervention. ${ }^{15-18}$ Previously, IAP antagonists, binding to selected BIR domains of IAPS and leading to cell death sensitization were designed. ${ }^{16,19-21}$ Recently, a new class of molecules mimicking the $\mathrm{N}$-terminal domain of SMAC (SMAC-mimetics) was developed, which bivalently bind to both BIR2 and BIR3 domains, and possess additional intrinsic apoptogenic activity via a TNF- $a$ feed forward loop. ${ }^{22,23}$ Within this TNF- $a$ loop, SMAC-mimetics stimulate the E3-ubiquitine ligase activity of clAPs, leading to autoubiquitination and subsequent proteasomal degradation of clAPs. Depletion of clAPs enables accumulation of NF- $k \mathrm{~B}$-inducing kinase (NIK), resulting in non-canonical activation of NF- $\mathrm{BB}$ and NF- $\mathrm{BB}$ target gene expression, including TNFA, which stimulates TNFR1 in an autocrine loop. ${ }^{23}$ In the absence of clAP, TNFR1stimulation triggers the assembly of the secondary RIP1containing cytoplasmatic complex (complex II), leading to caspase-8 activation and cell death induction. ${ }^{24}$ SMACmimetics may induce cell death dependently or independently of TNF- $a,{ }^{25-27}$ and we previously showed, that counteracting caspase inhibition by IAP antagonists sensitizes cancer cells for death receptor ligand- and chemotherapy-induced apoptosis. $^{28,29}$

Here, we analyze the therapeutic potential of the SMACmimetic BV6 in pediatric B-cell precursor (BCP)-ALL in vitro, ex vivo and in a preclinical model in vivo, and define the molecular requirements for intrinsic activity.

\section{Results}

Heterogeneous BV6 sensitivity of BCP-ALL cell lines. We assessed cell death induction upon exposure of the SMAC-mimetic BV6 in four BCP-ALL cell lines (UoCB6, REH, Nalm-6 and RS4;11). In two of the cell lines, BV6 induced cell death at nanomolar concentrations (half-maximal inhibitory concentration, IC ${ }_{50}$ UoCB6: $66.1 \mathrm{nM}$; IC $\left.{ }_{50} \mathrm{REH}: 251.1 \mathrm{nM}\right)$. In contrast, Nalm-6 and RS4;11 were resistant at concentrations in this nanomolar range beginning to show sensitivity for BV6 at clearly higher micromolar concentrations (Figure 1a). Nonmalignant lymphocytes (PBLs) obtained from healthy donors were largely refractory to BV6-induced cell death at nanomolar up to micromolar concentrations, demonstrating that BV6 is non-toxic to normal PBLs at concentrations sufficient to induce apoptosis in BV6-sensitive leukemia cells (Supplementary Figure 1A).

To address the mode of BV6-induced cell death, BCP-ALL cell lines were pretreated with the pan-caspase-inhibitor zVAD.fmk and the necrosis-inhibitor Necrostatin-1 (Nec-1). BV6-induced cell death was completely blocked by the pancaspase-inhibitor zVAD.fmk in sensitive cell lines indicating apoptosis as the primary cell death mechanism in these cells (Figure $1 \mathrm{~b}$ and $\mathrm{c}$ ). In contrast, cell death induced by high concentrations of BV6 in Nalm- 6 cells was only reduced to a minor extent by zVAD.fmk (Figure 1d), and zVAD.fmk induced even more cell death in RS4;11 cells (Figure 1e). Nec-1 had no inhibitory effect on resistant cell lines and only a minimal inhibitory effect on sensitive cell lines (Figure $1 b$ and e), also upon additional incubation with zVAD.fmk (Supplementary Figure $1 \mathrm{~B}$ and $\mathrm{C}$ ), indicating the absence of necrotic or necroptotic cell death.

SMAC-mimetics have been reported to induce cell death via a TNF-a loop. Interestingly, the TNFR fusion protein etanercept inhibited BV6-induced cell death in sensitive cell lines and to some extent in Nalm- 6 but not in RS4;11 cells, suggesting involvement of TNF- $a$ in cells sensitive to BV6-mediated apoptosis (Figure $1 \mathrm{~b}$ to $\mathrm{e}$ ).

Thus, we observed heterogeneous sensitivities toward the SMAC-mimetic BV6, which effectively induces TNF- $a$-dependent apoptosis at nanomolar concentrations in sensitive BCP-ALL cell lines, but importantly not in non-malignant, healthy lymphocytes.

BV6 induces an autocrine TNF- $a$ loop in BCP-ALL cell lines. Next, we investigated involvement of TNF- $a$ in molecular detail. SMAC-mimetics have been reported to trigger autoubiquitination and proteasomal degradation of cIAPs, NIK accumulation and NF- $K B$ activation followed by TNF- $a$ production and secretion. Interestingly, BV6 caused rapid clAP1 degradation in all cell lines (Figure 2a; Supplementary Figure 3). However, in contrast to clAP1, clAP2 is hardly detectable in BCP-ALL cell lines and was not affected by BV6 (Supplementary Figure 2A and B). Subsequent to clAP1 degradation, we observed increasing NIK levels, indicating an accumulation of NIK (Figure 2b; Supplementary Figure 3A and B). In line, a decrease in p100 and an increase in p52 were observed, pointing to activation of the non-canonical NF- $\kappa \mathrm{B}$ pathway. Moreover, increasing plkB levels indicated activation of the canonical NF- $\kappa \mathrm{B}$ pathway (Figure 2c; Supplementary Figure 3C). Interestingly, TNF- $a$ secretion was induced in BV6-sensitive cell lines, but to a lesser extend or not in BV6-insensitive cells (Figure 2d).

BV6 activates apoptosis signaling pathways in a TNF- $a$-dependent manner, mediated by the TNFR complex II. In order to analyze whether the TNFR complex II is required for BV6-mediated cell death induction, complex formation (caspase-8, RIP1 and FADD) was investigated.

Importantly, caspase-8 immunoprecipitation revealed marked increase of the TNFR complex II in BV6-sensitive cells (Figure $2 \mathrm{e}$ and h; Supplementary Figure 3D and G). Addition of etanercept attenuated this complex formation, consistent with the requirement of secreted TNF- $a$ in this model of apoptosis (Figure $2 e$ and $\mathrm{f}$ ).

Next, we evaluated cell death signaling downstream of the TNFR complex II. In contrast to resistant cells, a significant loss of mitochondrial membrane potential, a constant feature of type-II cells and largely observed in ALL, was detected upon BV6 in sensitive ALL cell lines, which was clearly reduced by etanercept, indicating activation of the intrinsic apoptosis pathway downstream of TNFR complex II (Figure 3a). Caspase-3 activation with decrease in proforms and increase in cleavage products was predominantly seen in the BV6-sensitive cell lines (Figure 3b; Supplementary Figure 4A) and almost completely inhibited by etanercept (Figure $3 c$; 

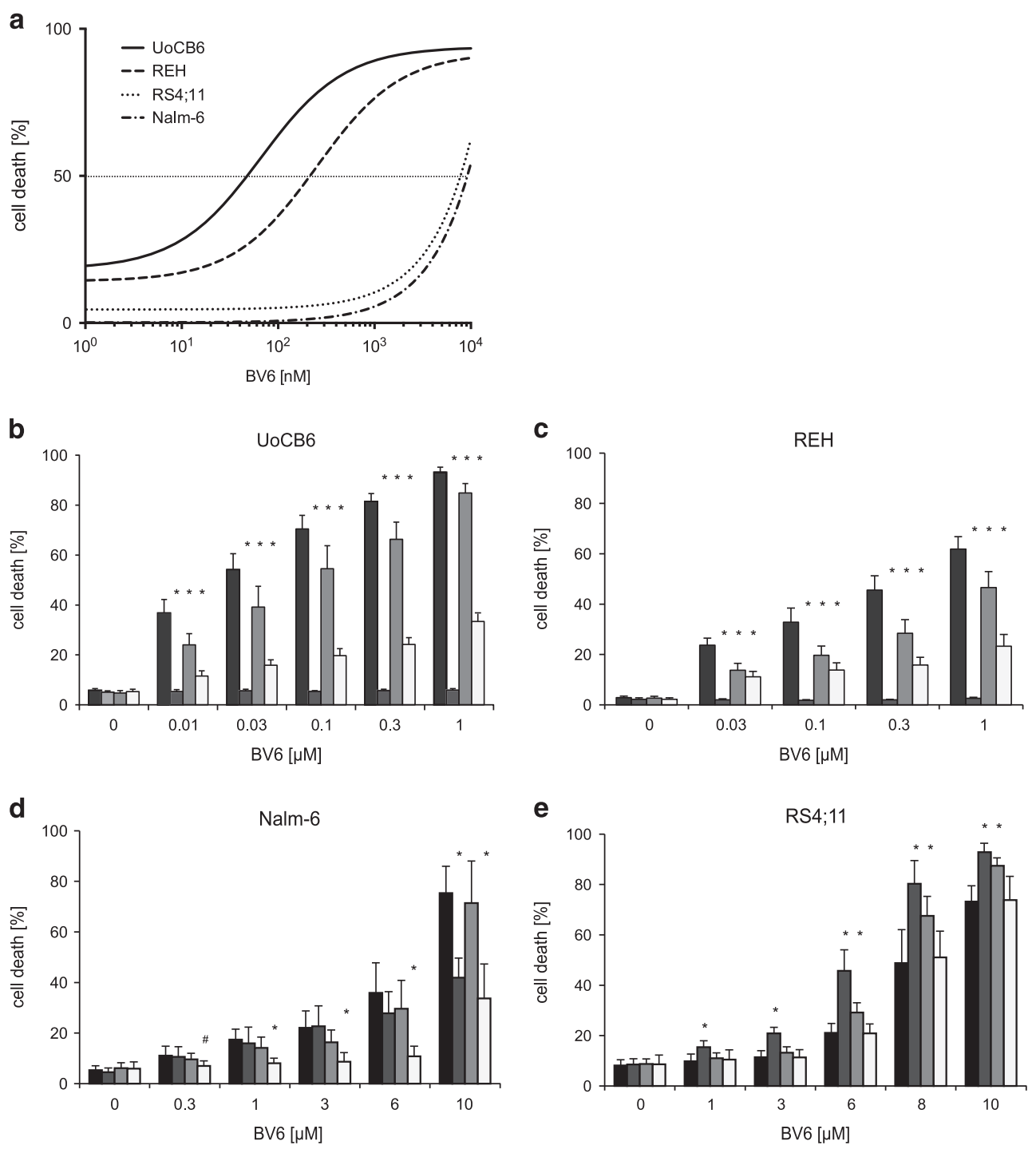

Figure 1 Intrinsic activity of BV6 on BCP-ALL cell lines. (a) Heterogeneous sensitivity of BCP-ALL cell lines for cell death after exposure to BV6 (48 h). UoCB6 and REH display half-maximal inhibitory concentration $\left(\mathrm{IC}_{50}\right)$ values at nanomolar concentrations in contrast to Nalm-6 and RS4;11 showing sensitivity in the micromolar range. (b-e) Inhibition of BV6-induced cell death (48-h exposure to BV6 at indicated concentrations) by $20 \mu \mathrm{M}$ zVAD.fmk (zVAD), $30 \mu \mathrm{M}$ Necrostatin-1 (Nec-1), or $40 \mu \mathrm{g} / \mathrm{ml}$ etanercept (Et). Percentages of dead cells were estimated by flow cytometry according to forward and side scatter criteria, three independent experiments each performed in triplicate, estimation of $\mathrm{IC}_{50}(\mathbf{a})$, comparison of $\mathrm{BV} 6$ to BV6 and the respective inhibitors; mean and S.D. are indicated; significance by Mann-Whitney $U$-test; ${ }^{*} P<0.01$ and ${ }^{\#} P<0.05$ (b-e)

Supplementary Figure 4B), indicating TNF-a-dependent caspase activation downstream of the TNFR complex II. Consistently, PARP was cleaved in sensitive but not in resistant cell lines (Figure 3d; Supplementary Figure 4C). These findings highlight the essential requirement of the TNFR complex for BV6-induced activation of cell death signaling.

Apoptosis induction by BV6 is dependent on RIP1. clAPs have been reported to modulate RIP1 by ubiquitination with their E3 ligase activity, thereby determining the function of RIP1 in the TNFR complex. Therefore, we explored the role of RIP1 in TNFR complex II-mediated apoptosis induction upon BV6 treatment. RNA interference-mediated knockdown of
RIP1 reduced SMAC-mimetic-induced cell death clearly in BV6-sensitive cell lines (Figure $4 a$ and b), whereas this effect was not observed in BV6-insensitive cell lines (Figure 4c and d). Reduced RIP1 protein expression upon siRNA-mediated knockdown was confirmed by western blot analyses (Supplementary Figure 5A and D).

To further elucidate the role of RIP1, we analyzed downstream apoptosis signaling in the presence or absence of RIP1 in the BV6-sensitive cell line REH. REH cells with stable shRNA-mediated RIP1 knockdown showed a clear reduction of cell death (Figure 4e), blocked activation of intrinsic and extrinsic apoptosis signaling as indicated by absence of loss of mitochondrial membrane potential (Figure 4f) and lack of caspase activation (Figure 4h; 

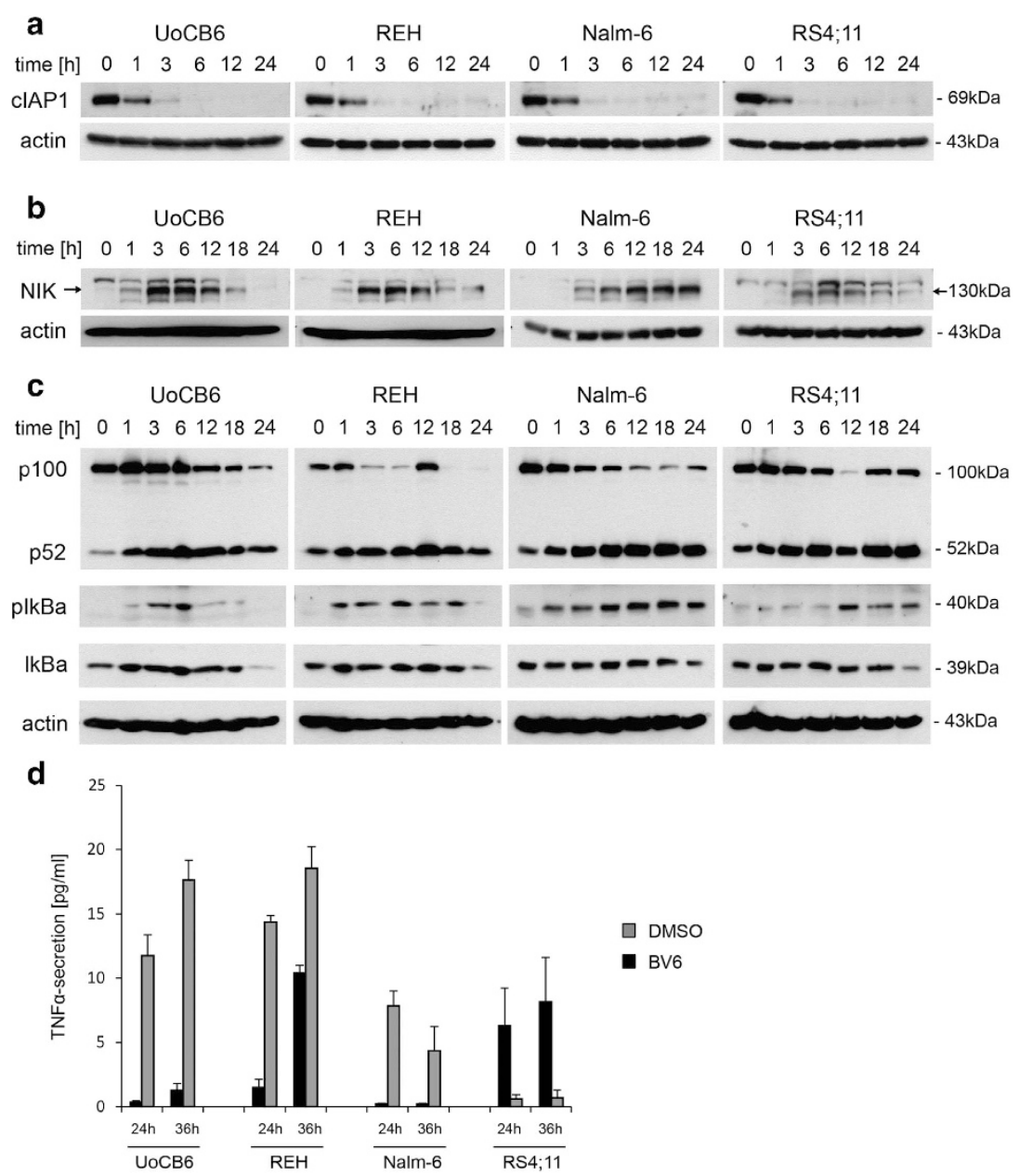

e

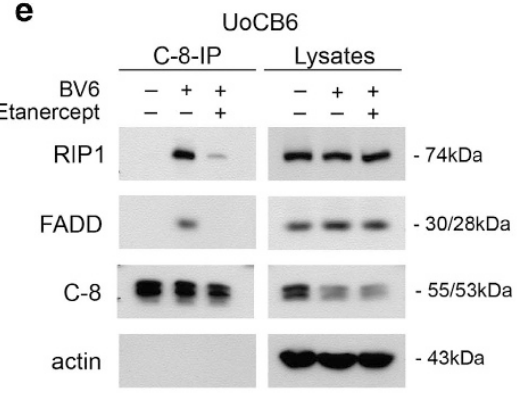

g

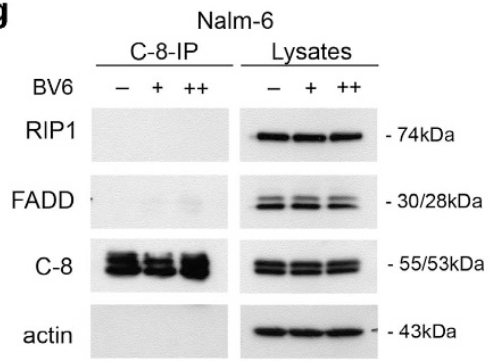

f

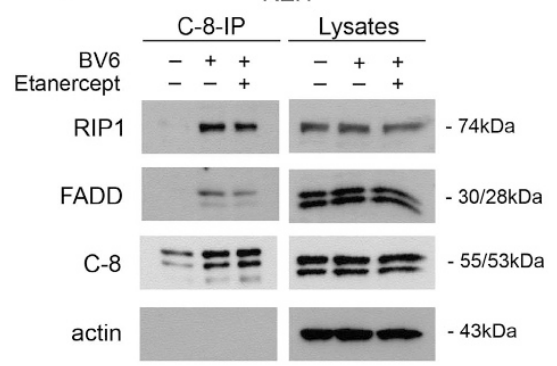

h

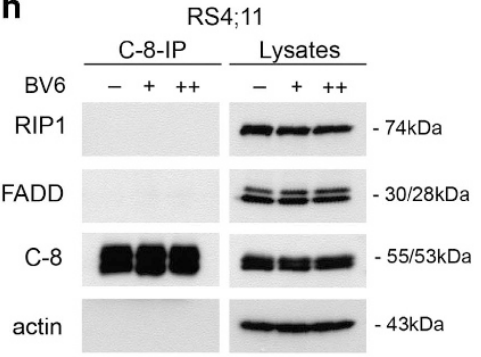

Figure 2 TNF- $\alpha$ signaling upon BV6 exposure. Western blot analysis of (a) cIAP1 degradation, (b) NIK accumulation and (c) NF- $\kappa$ B activation in BCP-ALL cell lines exposed to BV6 for indicated time points (UoCB6 and REH: $1 \mu \mathrm{M} \mathrm{BV6}$; Nalm-6 and RS4;11: $10 \mu \mathrm{M} \mathrm{BV6}$; or equivalent doses of DMSO for $24 \mathrm{~h}$ ). For each analysis, one representative blot of three is shown. Arrows indicate NIK. (d) Increased TNF- $\alpha$ protein levels in cellular supernatants of BCP-ALL cell lines treated with BV6 or control as indicated (mean and S.D. of three independent experiments each performed in duplicate). (e-h) TNFR complex II formation upon BV6 exposure (UoCB6 and REH: $1 \mu \mathrm{M} ; \mathrm{Nalm}-6$ and RS4;11: $1 \mu \mathrm{M}(+)$ or $10 \mu \mathrm{M}(++)$ BV6) and dependency on TNF- $\alpha$ (with or without $40 \mu \mathrm{g} / \mathrm{ml}$ etanercept). Caspase-8 immunoprecipitation in the presence of $10 \mu \mathrm{M}$ zVAD.fmk (UoCB6, REH and Nalm6), and western blot analysis of indicated proteins (one representative experiment of three is shown) 

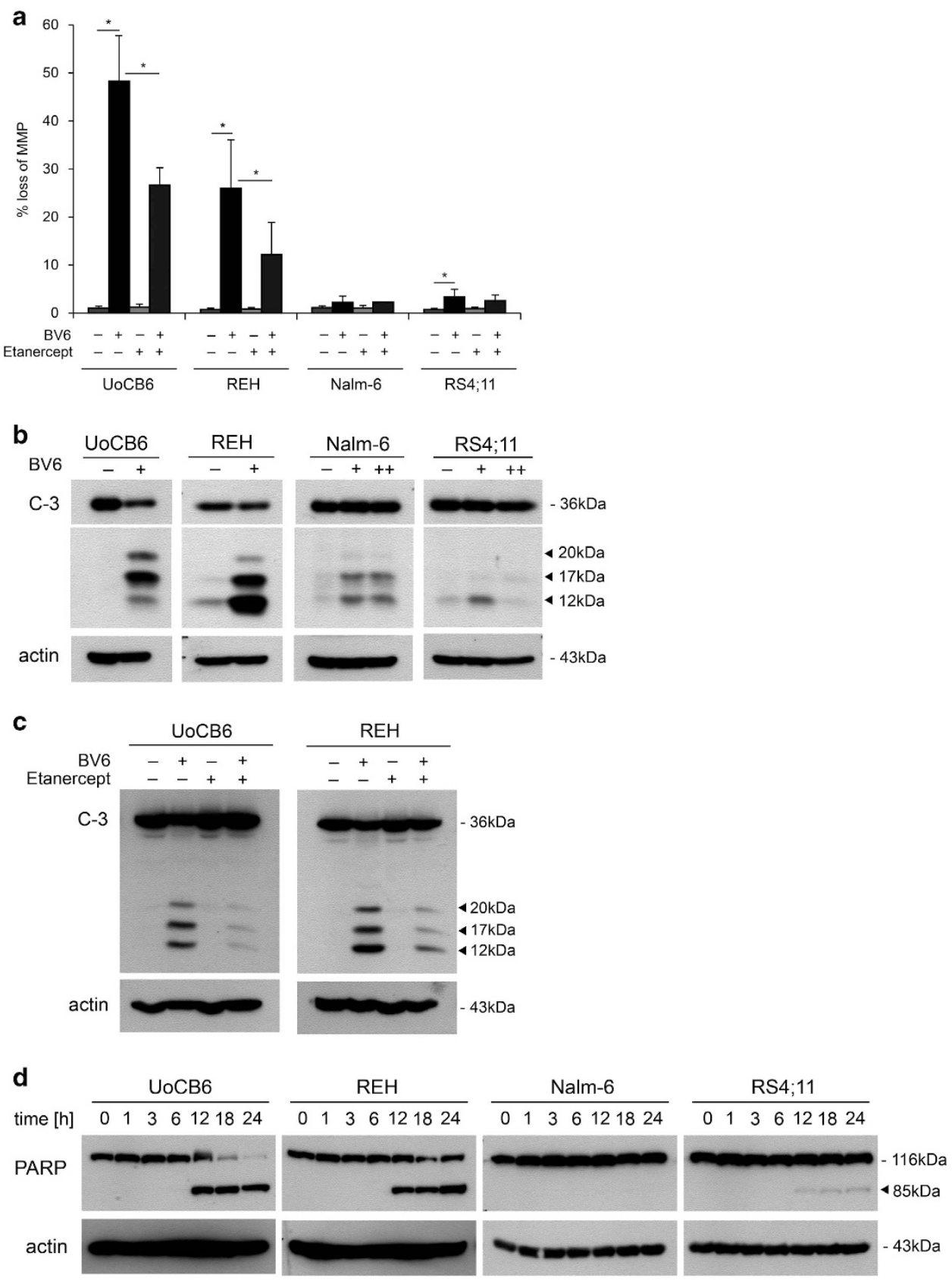

Figure 3 Apoptosis signaling upon BV6 treatment. (a) Loss of mitochondrial membrane potential (MMP) in BCP-ALL cell lines upon BV6 treatment (UoCB6 and REH: $1 \mu$ M;

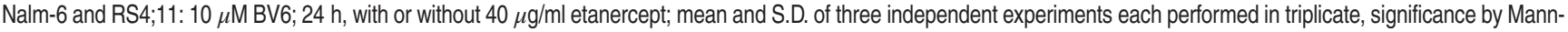
Whitney U-test, ${ }^{*} P<0.01$. (b) Caspase-3 activation in BCP-ALL cell lines upon BV6 treatment (UoCB6 and REH: $1 \mu \mathrm{M}$; Nalm-6 and RS4;11: $1 \mu \mathrm{M}(+)$ or $10 \mu \mathrm{M}$ (++) BV6; 24 h), and (c) TNF- $\alpha$ dependency of the BV6-sensitive leukemia cell lines UoCB6 and REH (additional treatment with or without $40 \mu \mathrm{g} / \mathrm{ml}$ etanercept), western blot analysis. (d) PARP cleavage upon BV6 treatment for indicated time points (UoCB6 and REH: $1 \mu \mathrm{M}$; Nalm-6 and RS4;11: $10 \mu \mathrm{M}$ BV6), western blot analysis. For each western blot analysis, one representative experiment out of three is displayed

Supplementary Figure 5E). Moreover, caspase-8 immunoprecipitation showed a profound reduction of FADD recruitment in the absence of RIP1 (Figure 4i; Supplementary Figure 5F), demonstrating that RIP1 is required for complex II formation.

Taken together, these data show that apoptosis induction by the SMAC-mimetic BV6 in BCP-ALL is dependent on RIP1.
BV6 induces cell death in primograft leukemia samples dependent on TNF- $a$ including high-risk and poor outcome ALL. Next, we investigated the effects of BV6 in a series of 40 primograft ALL samples established in our NOD/ SCID/huALL mouse model by xenografting therapy-naive patient ALL cells obtained at first diagnosis or relapse of pediatric BCP-ALL patients (Table 1). Cell death induction was observed in the majority of leukemias with two-thirds of 
primografts showing induction of $25 \%$ or more cell death upon exposure to BV6 (Figure 5A).

In a subset of 20 samples, etanercept inhibited BV6-mediated cell death indicating TNF- $a$ dependency also in patient-derived ALL primografts as observed in sensitive cell lines (Figure 5B).

However, no association of leukemia or patient characteristics and clinical outcome with BV6 sensitivity was observed.
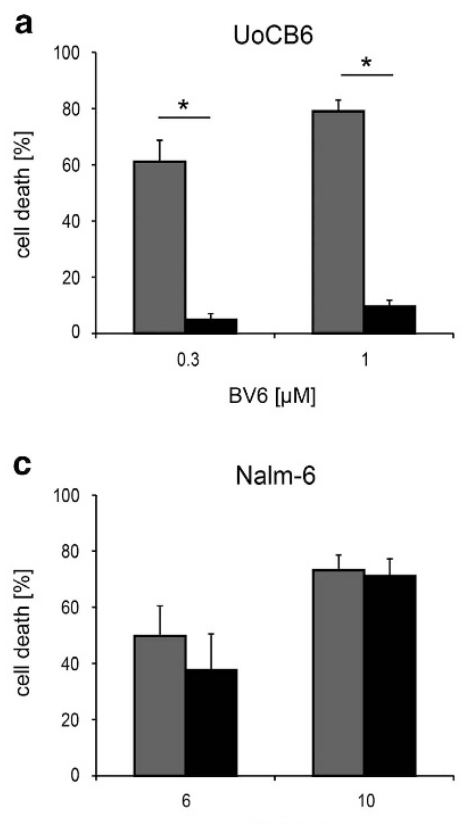

BV6 $[\mu \mathrm{M}]$

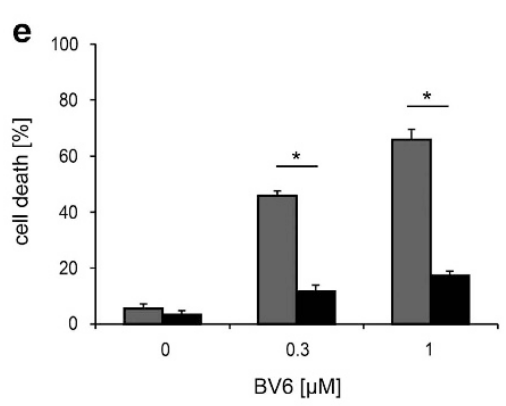

h

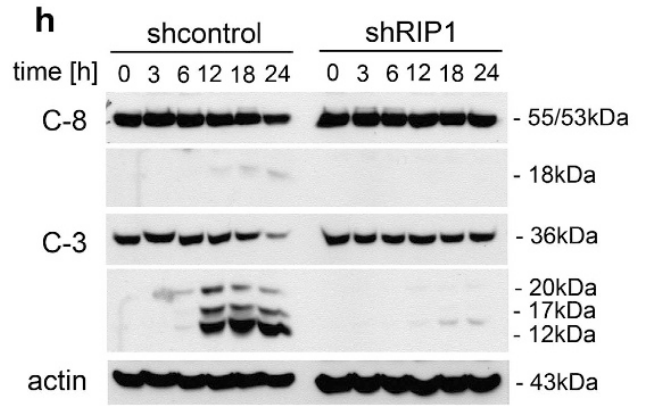

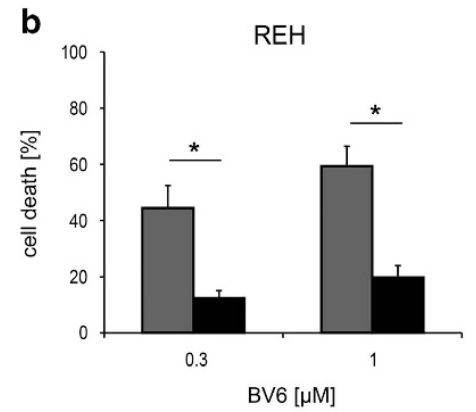

d

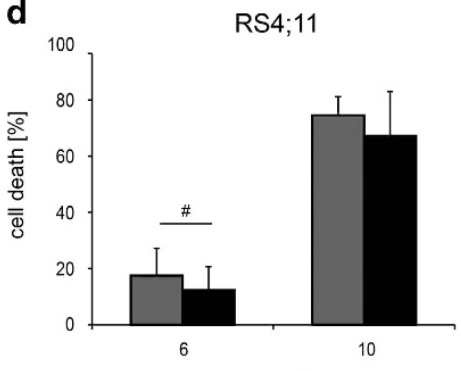

BV6 $[\mu \mathrm{M}]$
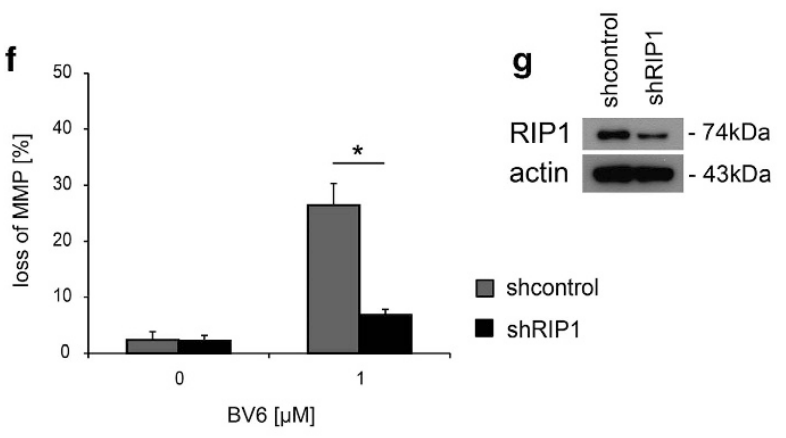

shcontrol

shRIP1

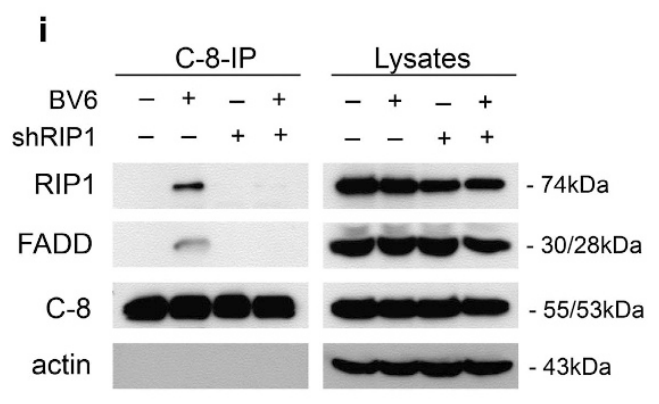

Figure 4 Dependency of BV6-induced cell death on RIP1. Block of BV6-induced cell death (exposure at indicated concentrations for 48 h) by RIP1 knockdown in BV6sensitive ( $\mathbf{a}$ and $\mathbf{b}$ ) but not BV6-insensitive ( $\mathbf{c}$ and $\mathbf{d}$ ) cell lines. Mean and S.D. of three independent experiments each performed in triplicate are shown, significance by MannWhitney U-test; ${ }^{*} P<0.01$ and ${ }^{\#} P<0.05$. (e) Blocked BV6-induced cell death (48-h exposure at indicated concentrations), and (f) loss of mitochondrial membrane potential (MMP; $24 \mathrm{~h}, 1 \mu \mathrm{M} \mathrm{BV6}$ ) in REH cells with stable shRNA-mediated RIP1 knockdown. Mean and S.D. of three independent experiments each performed in triplicate are shown, significance by Mann-Whitney U-test; ${ }^{*} P<0.01$. (g) Efficient knockdown of RIP1 in shRNA versus control-transduced REH cells is shown by western blot analysis. (h) Absence of caspase- 8 and -3 activation ( $1 \mu \mathrm{M} \mathrm{BV} 6$ for indicated time points) in REH cells upon shRNA-mediated RIP1 knockdown, as well as (i) impaired complex II formation ( $1 \mu \mathrm{M}$ BV6 for $18 \mathrm{~h}$, caspase- 8 immunoprecipitation in the presence of $10 \mu \mathrm{M}$ zVAD.fmk and western blot analysis of indicated proteins) 
Table 1 Characteristics of patients and derived primograft leukemia samples

\begin{tabular}{|c|c|c|}
\hline & $\mathbf{N}$ & $\%$ \\
\hline Total & 40 & 100 \\
\hline \multicolumn{3}{|l|}{ Primograft derived from } \\
\hline Initial diagnosis & 37 & 93 \\
\hline Relapse & 3 & 7 \\
\hline \multicolumn{3}{|l|}{ Gender } \\
\hline Male & 23 & 58 \\
\hline Female & 17 & 42 \\
\hline \multicolumn{3}{|l|}{ Age (years) } \\
\hline $1-9$ & 27 & 68 \\
\hline$<1$ or $>9$ & 13 & 32 \\
\hline \multicolumn{3}{|l|}{ Immunophenotype } \\
\hline Pro-B ALL & 4 & 10 \\
\hline C-ALL & 26 & 65 \\
\hline Pre-B ALL & 10 & 25 \\
\hline \multicolumn{3}{|l|}{ Gene alterations } \\
\hline ETV6/RUNX1 & 7 & 17 \\
\hline$B C R / A B L$ & 1 & 3 \\
\hline$M L L$-rearrangement & 4 & 10 \\
\hline None of the above & 28 & 70 \\
\hline \multicolumn{3}{|l|}{ BFM high risk } \\
\hline Yes & 5 & 13 \\
\hline No & 32 & 80 \\
\hline Not applicable & 3 & 7 \\
\hline \multicolumn{3}{|l|}{ Prednisone response } \\
\hline Poor & 1 & 3 \\
\hline Good & 36 & 90 \\
\hline Not applicable & 3 & 7 \\
\hline \multicolumn{3}{|c|}{ Resistance to spontaneous apoptosis } \\
\hline Yes & 5 & 13 \\
\hline No & 11 & 27 \\
\hline Not analyzed & 24 & 60 \\
\hline \multicolumn{3}{|l|}{ Time to leukmia (TTL) } \\
\hline Short & 11 & 27 \\
\hline Long & 29 & 73 \\
\hline
\end{tabular}

Interestingly, leukemia samples derived from BFM-high-risk stratified patients (\#11, \#12, \#21, \#23 and \#27) or from patients at relapse (\#4, \#35 and \#39) were sensitive to BV6. Previously, we observed that rapid engraftment of patientderived ALL cells transplanted onto NOD/SCID mice (short time to leukemia, $\mathrm{TTL}^{\text {short }}$ ) indicates early patient relapse. ${ }^{8}$ Of note, BV6 sensitivity was also seen in samples of this poor-prognosis phenotype (\#6, \#8, \#11, \#22, \#23, \#24, \#27, \#28, \#31, \#33 and \#34). Moreover, we described earlier that deficient spontaneous apoptosis signaling in patient and xenograft BCP-ALL cells is associated with poor patient outcome. ${ }^{7,9}$ By the same strategy, 5 of 16 samples investigated showed intrinsic resistance to spontaneous apoptosis (\#6, \#11, \#22, \#31 and \#38), of which four were BV6-sensitive. Furthermore, addressing key downstream apoptosis signaling events, we identified mitochondrial cytochrome $c$ release and activation of caspase-3, indicating BV6-induced activation of apoptosis signaling in primary BCP-ALL (Figure 5Ca and b).

In summary, SMAC-mimetics induce clear activity in the majority of patient-derived ALL samples, and importantly in leukemias associated with high-risk characteristics, poor patient outcome, relapse and apoptosis deficiency.

BV6 treatment is highly effective in a preclinical NOD/ SCID/huALL mouse model in vivo. On the basis of these results, we further evaluated the efficacy of BV6 in a preclinical in vivo setting on a primograft leukemia sample with rapid NOD/SCID engraftment, and deficient apoptosis signaling derived from a patient who later on suffered from early relapse (\#31). Recipient animals were transplanted, and upon leukemia manifestation treated with either BV6 or solvent (10 recipients per group) for 2 weeks. The medication was well tolerated and no signs of in vivo toxicity were observed. Interestingly, a significant reduction of leukemia load at the end of therapy, a significant delay of disease reoccurrence and prolonged leukemia-free survival were observed upon BV6 in vivo treatment as compared to control-treated animals (Figure 6a to $\mathrm{c}$ ).

In a clinical setting, however, high-risk disease is unlikely to be treated by one compound alone. Therefore, we combined BV6 with multidrug chemotherapy resembling ALL induction treatment and observed an almost complete reduction of tumor load, a significant delay of leukemia reoccurrence and prolonged survival (Figure 6a, b and d) of animals treated with the combination of SMAC-mimetic and chemotherapy compared to chemotherapy alone.

Importantly, additional administration of etanercept reversed the in vivo effect of BV6 single treatment on leukemia-free survival, indicating TNF- $a$ dependency also in vivo (Figure 6e). Etanercept on its own, however, did not affect leukemia-free survival (Figure 6e). In contrast, abrogation of TNF- $a$ signaling had only a minor effect on survival after combination therapy with VDA and BV6, suggesting that the sensitizing effect of BV6 for chemotherapy is only partially TNF- $a$ dependent (Figure 6f).

\section{Discussion}

IAPs have been shown to be overexpressed in several cancers including acute leukemia, and to be associated with treatment failure and inferior prognosis. ${ }^{17,18}$ In line with the prognostic role of intact cell death pathways, we found that deficient apoptosis signaling in primary BCP-ALL cells is indicative of early patient relapse. ${ }^{7-10}$ Thus, antagonizing IAP proteins by, for example, SMAC-mimetics may represent an attractive strategy to activate the apoptotic machinery and to overcome resistance. In previous studies, we and others have shown that IAP inhibition sensitizes various tumor cells, including acute leukemia cells, for cell death-inducing stimuli in vitro and in vivo. ${ }^{28,29}$ Also, single agent activities of IAP inhibitors and small-molecule SMAC-mimetics have been reported for several tumor cell lines, and some primary tumor samples including AML and CLL. ${ }^{30,31}$ However, the spectrum of activity of SMAC-mimetics in ALL has not been examined in detail so far.

Here we demonstrate, that the small-molecule SMACmimetic BV6 is highly effective to induce cell death in ALL cell lines and, most importantly, in a wide range of patientderived primograft samples, and is effective on high-risk 

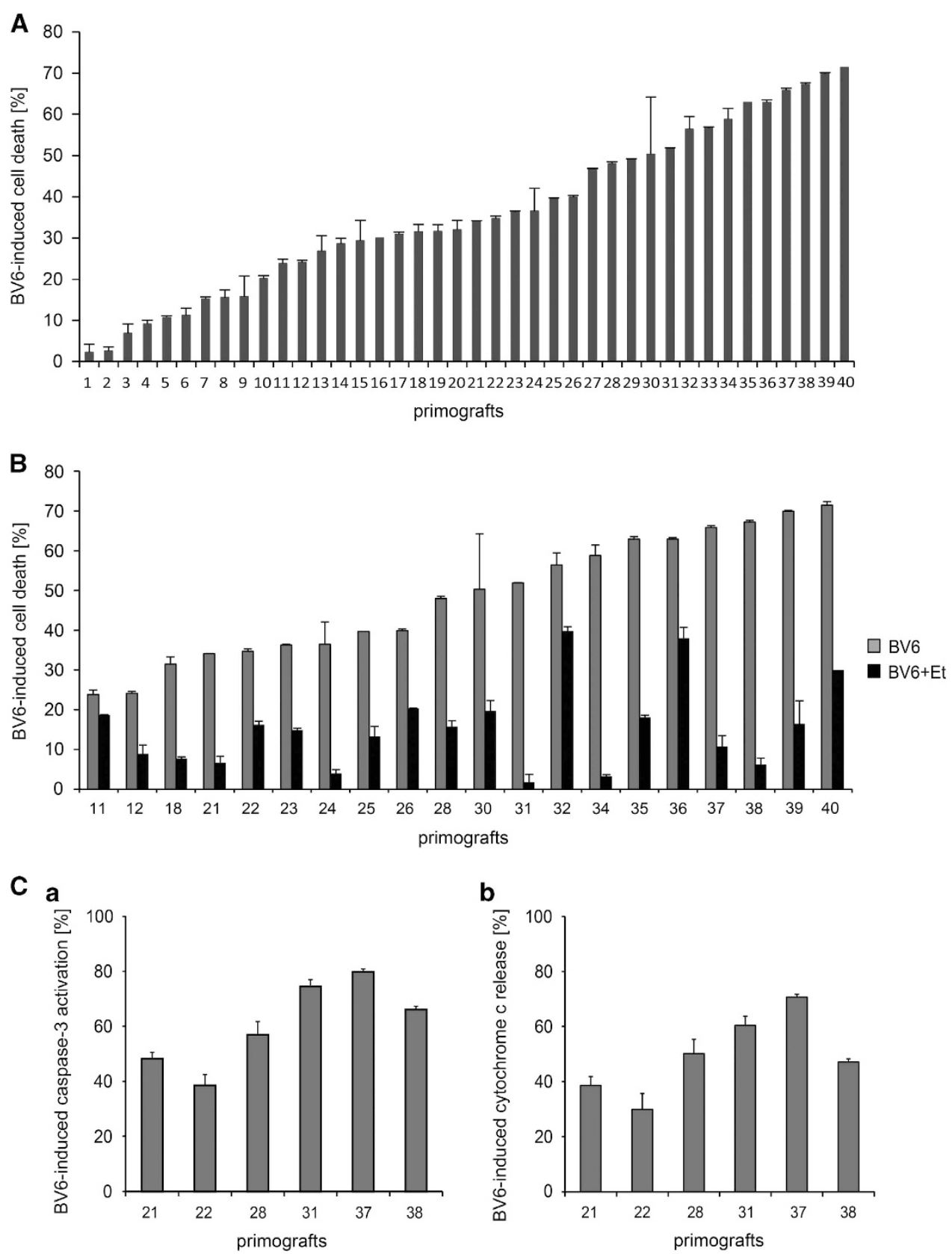

Figure 5 BV6-induced cell death in primary ALL. Cell death induction in primary, patient-derived BCP-ALL samples $(n=40)$ by BV6 (A) and dependency on TNF- $\alpha(n=20$ primografts; B). Cell death by flow cytometry according to forward and side scatter criteria upon treatment with $1 \mu \mathrm{M} \mathrm{BV6}$ for $48 \mathrm{~h}$ and with $40 \mu \mathrm{g} / \mathrm{ml}$ (BV6+Et) or without, BV6induced cell death was calculated as the difference of total and spontaneous cell death; experiments performed in triplicate; mean and S.D. are indicated. (C) Activation of caspase-3 (a), and cytochrome $c$ release (b) upon BV6 treatment $(1 \mu \mathrm{M}, 18 \mathrm{~h})$ in primograft samples. Percentages of cells with activated caspase-3 and cells with released cytochrome $c$ were estimated by flow cytometry in triplicate, bars represent mean values and S.D.

leukemia leading to prolonged survival in a preclinical NOD/SCID/huALL primograft model in vivo.

Interestingly, we detected a concentration-dependent sensitivity to BV6 in BCP-ALL cell lines. Cell lines sensitive for lowdose BV6 (UoCB6 and REH) exhibited a full activation of the TNF- $a$ feed forward loop: rapid degradation of cIAP, NIK stabilization, NF- $\kappa$ B activation and TNF- $a$ secretion, followed by TNF- $\alpha$-dependent apoptosis induction, as demonstrated by addition of the soluble TNFR etanercept, which prevented mitochondrial perturbation, caspase activation and apoptosis. Furthermore, we found that TNF- $a$ is secreted by ALL cells and subsequently triggers the formation of the TNFR complex II consisting of caspase-8, RIP1 and FADD. This complex was described to be formed as a result of clAP degradation by SMAC-mimetics at the stimulated TNFR1 in the presence of de-ubiquitinated RIP1. ${ }^{24,32}$ In line with this, we found formation of TNFR complex II upon cIAP1 degradation and that complex formation is dependent on TNF- $a$, since the addition of 

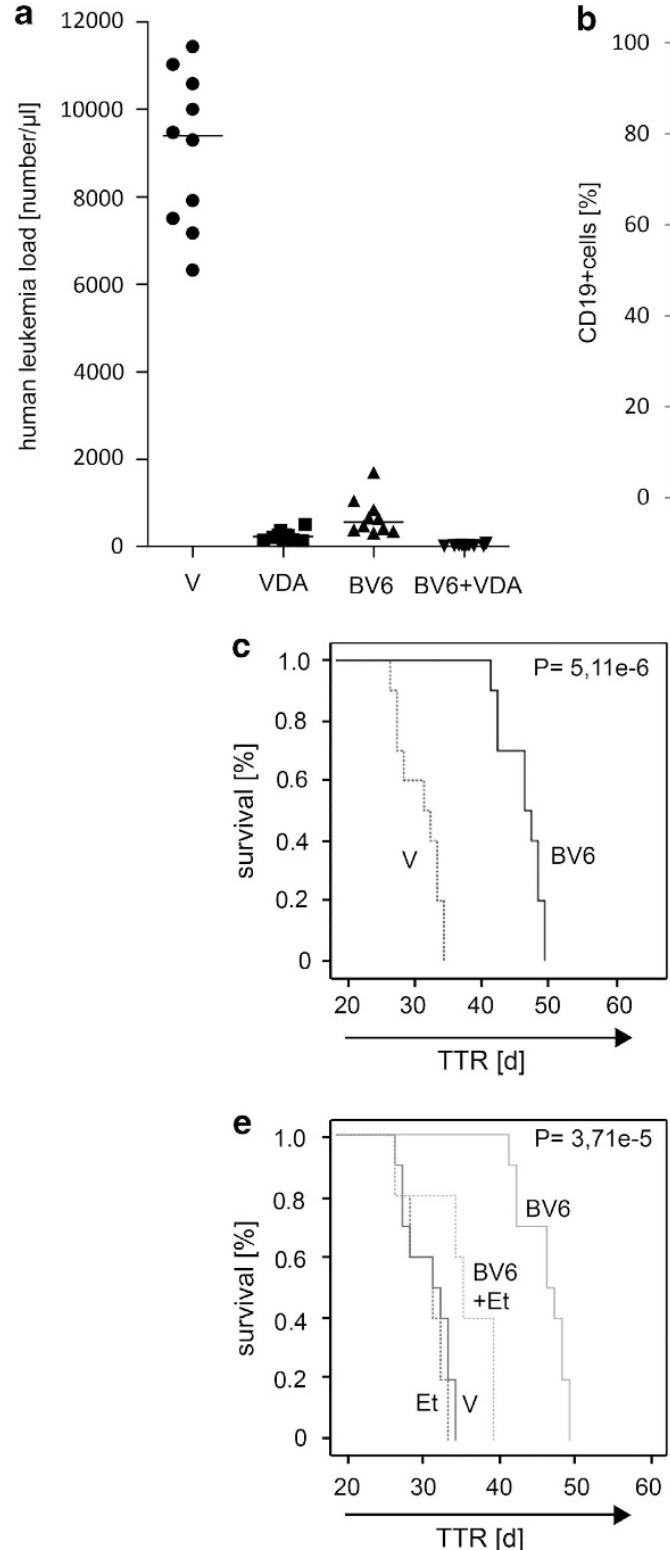

b
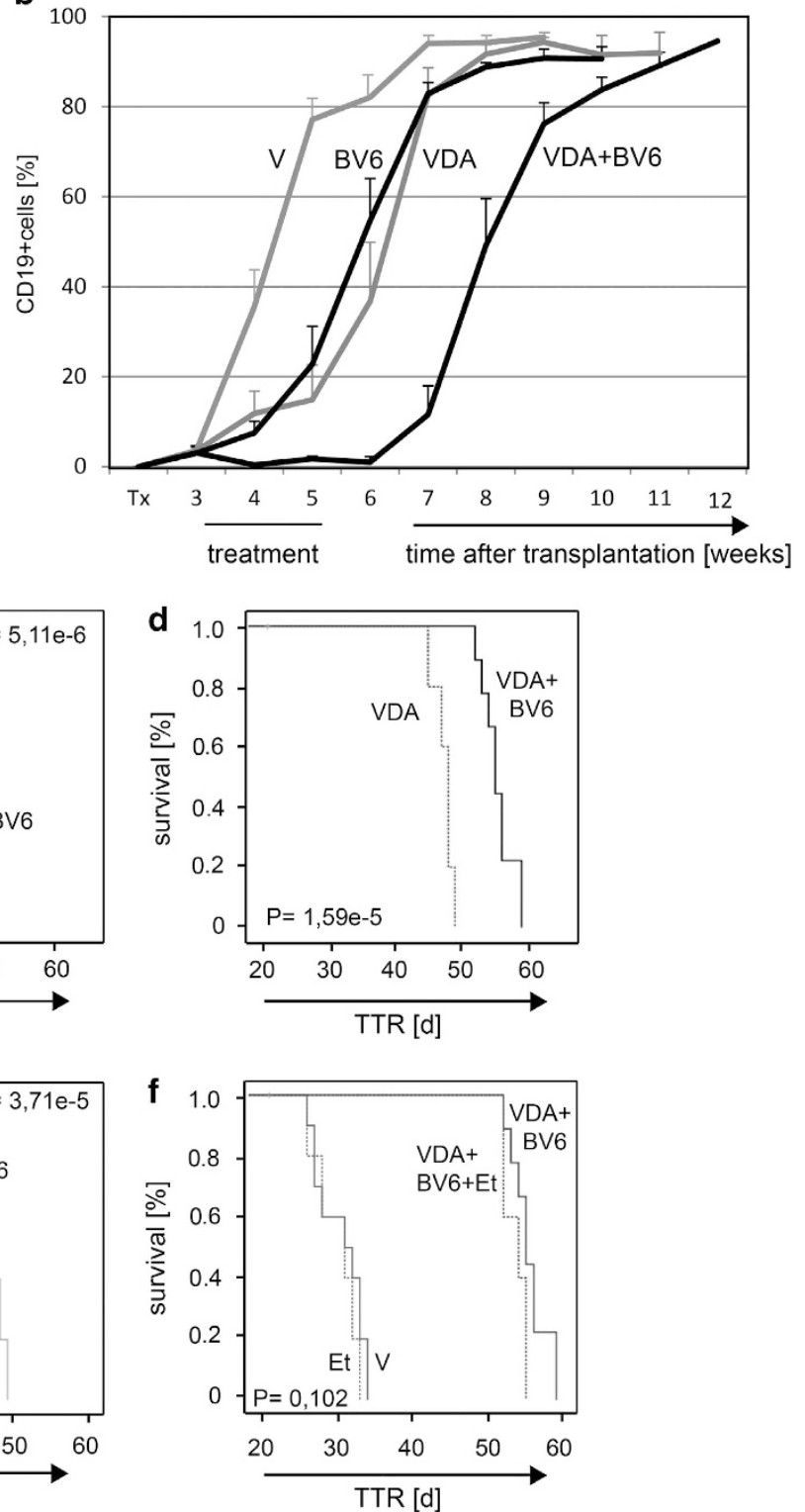

Figure 6 Preclinical activity of BV6 on pediatric high-risk ALL. NOD/SCID mice transplanted with a high-risk BCP-ALL showing presence of human ALL cells in the peripheral blood were treated as indicated. (a) Leukemia load (numbers of human leukemia cells in the recipient's peripheral blood at the end of treatment, recipients individual and median values are shown). (b) Percentages of human (CD19 positive) ALL cells in the recipient's peripheral blood over time (data points represent mean values and S.D. per group). Superior leukemia-free survival of ALL-bearing animals after treatment with BV6 (c), a combination of VDA induction-chemotherapy and BV6 compared to chemotherapy alone (d), and in vivo TNF- $\alpha$ dependency of intrinsic (e) but not of chemo-sensitizing BV6 activity (f). Probabilities of leukemia-free survival after treatment with vehicle (V), BV6, induction-chemotherapy (vincristine, dexamethasone and asparaginase; VDA) or combinations thereof (10 animals per group) with or without etanercept (Et; five animals per group); TTR, time to leukemia reoccurrence; Kaplan-Meier analysis, log-rank test; $P$, significance

etanercept reduced complex formation. Accordingly, exposure of the BCP-ALL cell line REH to etanercept was reported to decrease BV6-mediated cell death; however, this effect was not observed in the T-ALL line Jurkat. ${ }^{33}$ Furthermore, we provide evidence that the presence of RIP1 is crucial for apoptosis induction upon BV6 treatment. Thus, knockdown of RIP1 abrogated the formation of complex II and profoundly reduced SMAC-mimetic-induced mitochondrial perturbation, caspase activation and apoptosis induction. However, inhibition of the RIP1 kinase by Nec-1 had only a slight effect on apoptosis induction, demonstrating that cell death induction by SMAC-mimetics is dependent on the availability of RIP1 in the complex but not on the kinase activity of RIP1. Although cell lines resistant to BV6 (Nalm-6 and RS4;11) showed rapid cIAP degradation, NIK stabilization and NF- $\kappa \mathrm{B}$ activation after BV6 treatment, lower or no enhanced TNF- $a$ production was detectable in these cells. The lack of BV6induced TNF production suggests that sensitivity to the 
intrinsic activity of SMAC-mimetics at lower concentrations strictly depends on the TNF- $a$ loop. However, no associations of BV6-sensitivity and genetic aberrations including TP53 mutations were observed.

SMAC-mimetics have been reported to have low ex vivo activities on different cancer xenografts with a majority of tumors being resistant and a minority showing sensitivity to SMAC-mimetics mostly at higher micromolar concentrations. ${ }^{34,35}$ In AML and CLL, cells have been reported to be sensitive for SMAC-mimetics in single and combination treatment regimens ex vivo, predominantly also at micromolar concentrations. ${ }^{30,31}$ However, at these higher concentrations toxic effects on monocytes and dendritic cells have been observed. ${ }^{36}$ Recently, SMAC-mimetics have been shown to moderately sensitize primary ALL cells for dexamethasone ex vivo and in a non-systemic, subcutaneous leukemia model in vivo, ${ }^{33}$ and no in vivo intrinsic activity on ALL was observed for the SMAC-mimetic LCL-161. ${ }^{35}$

In contrast, we found that the majority of patient-derived ALL samples is sensitive for the SMAC-mimetic BV6, including leukemias with high-risk/poor prognostic features. Primograft samples, which were sensitive for BV6 showed TNF-a dependent cell death induction. Most importantly, we also found that BV6 treatment leads to activation of intrinsic and extrinsic apoptosis pathways, also in leukemias with deficient constitutive apoptosis signaling indicative for early patient relapse. Thus, SMAC-mimetics are effective in leukemias including ALL with high-risk characteristics and intrinsic apoptosis resistance.

Our study also provides clear evidence that the SMAC-mimetic BV6 is highly effective in a BCP-ALL primograft model in vivo. Single treatment with BV6 led to a significant reduction of tumor burden and prolonged leukemiafree survival. This intrinsic activity of BV6 was clearly dependent on TNF- $a$, since concomitant in vivo treatment with etanercept reversed the effect. The combination of BV6 with conventional chemotherapy was even more effective, resulting in substantial reduction of tumor load and significant delay of post-treatment leukemia reoccurrence. This effect, however, was largely independent of TNF-a suggesting that the sensitizing effect of SMAC-mimetics might be mainly due to the prevention of caspase inhibition.

In summary, SMAC-mimetics exhibit high-intrinsic activity in ALL cell lines and in the vast majority of primary ALL samples in a TNF- $\alpha$-dependent manner. BV6 is also highly effective on patient-derived leukemia samples, associated with high-risk/ poor-prognosis features leading to significantly prolonged relapse-free survival in a high-risk preclinical NOD/SCID/ huALL primograft model, both as single treatment and in combination with conventional chemotherapy. With this, our findings provide important evidence for the potential use of SMAC-mimetics in treatment strategies of childhood BCP-ALL. As SMAC-mimetics recently entered phase II clinical trials as single agents and in combination with chemotherapeutics, they may be deployable for clinical use shortly. Our studies on normal lymphocytes did not reveal any effect at concentrations sufficient to induce cell death in ALL cells, and most SMAC-mimetics have been reported to be well tolerated in phase I and II studies. ${ }^{37,38}$ Since SMAC-mimetics induce cell death and reactivate apoptosis signaling even in apoptosis-resistant cells of high-risk patients, they may be used in treatment protocols for high-risk/poor-prognosis ALL patients. Furthermore, the increased effect of SMAC-mimetics along with conventional chemotherapeutics offers the opportunity to use them also in standard-risk groups to reduce the dose of cytotoxic drugs, thereby decreasing chemotherapyrelated side effects. Our data also define the requirements for the intrinsic activity of BV6 and possibly also other SMAC-mimetics: induction of TNF- $a$ and availability of RIP1 in the TNFR complex. These may be used as biomarkers to select ALL patients for treatment with SMAC-mimetics such as BV6. Interestingly, in contrast to the TNF- $a$-dependent intrinsic activity of BV6, the chemotherapy-sensitizing effect appears to be mediated by the 'classical' SMAC function, that is, inhibition of IAPs. Thus, the chemo-sensitizing effect may be used in IAP-expressing leukemias.

In conclusion, SMAC-mimetics present a promising new therapeutic approach for pediatric ALL, which deserves further investigation in clinical trials.

\section{Material and Methods}

Cells. Cell lines were obtained from DSMZ, Braunschweig, Germany; UoCB6 cells were kindly provided by Dr. Rowley, Chicago, USA. Cells were mycoplasmanegative, and authenticated by single-tandem-repeat profiling and kept under standard conditions. ${ }^{39}$ All four cell lines are of a BCP immunophenotype (BCP-ALL), REH and UoCB6 were derived from ETV6/RUNX1- ${ }^{-}$-rearranged, RS4;11 from MLL AF4-rearranged leukemias. ${ }^{40}$ Analysis of the cell lines for TP53 mutations by denaturing high-performance liquid chromatography followed by Sanger sequencing of exons 4-1041 identified TP53 mutations in the cell lines REH and RS4;11 while UoCB6 and Nalm-6 were found to be TP53 wild type.

PBLs were isolated from healthy donors and cultured $\left(37^{\circ} \mathrm{C}, 5 \% \mathrm{CO}_{2}\right)$ in RPMI 1640 with $10 \%$ fetal calf serum (Conco, Wiesbaden, Germany), 10 mM HEPES, $2 \mathrm{mmol} / \mathrm{L}$-glutamine, 30 units (U)/ml hulL-2 (Biochrom, Berlin, Germany), $100 \mathrm{U} / \mathrm{ml}$ penicillin and $100 \mu \mathrm{g} / \mathrm{ml}$ streptomycin (Life Technologies, Frankfurt, Germany). Adhesed monocytes were removed, and lymphocytes were selected according to forward side scatter properties.

NOD/SCID/huALL. Primograft leukemias were established transplanting patient ALL cells onto female, 6-week-old NOD/SCID mice (NOD/LtSz-scid/scid, Charles River, Germany) as previously described., ${ }^{7,39}$ Patient samples were obtained after informed consent of patients and/or their legal guardians in accordance with the institution's ethical review board, and all animal experiments were approved by the appropriate authority (Regierungspräsidium Tübingen, TVANr. 1147). Upon leukemia onset, xenograft cells (minimum 90\% huCD19-positive cells) were isolated from spleens and used for ex vivo analyses or transplanted onto subsequent recipients. All together 40 available patient-derived BCP-ALL samples (obtained after in median 2.5 passages) have been included into this study. Patient and leukemia characteristics are summarized in Table 1. For in vivo treatment, ALL cells of primograft \#31 were re-transplanted. On the basis of survival times (log-rank test), sample size estimates had been calculated (UIm University, Institute of Epidemiology and Medical Biometry), and recipients were subdivided into groups of ten by consecutive numbering. Upon appearance of more than $5 \%$ human leukemia cells in peripheral blood, vehicle (DMSO); VDA (vincristine, $0.075 \mathrm{mg} / \mathrm{kg}$, once per week; dexamethasone, $2.5 \mathrm{mg} / \mathrm{kg}, 5$ days per week; asparaginase, $500 \mathrm{IU} / \mathrm{kg}$, 5 days per week); BV6 (10 mg/kg, twice per week); or combinations were applied by intraperitoneal (i.p.) injections (investigator is non-blinded) for 2 weeks. In addition, etanercept (10 mg/kg, i.p., twice per week, 2 weeks) or combinations with VDA/BV6 were applied (five mice per group). Human leukemia was monitored weekly in PB by flow cytometry staining for huCD19 (anti-CD19-Allophycocyanin, \#555415 and LSR-II cytometer, BD Bioscience, Heidelberg, Germany). White blood cell counts were performed once after completion of treatment. At onset of leukemia-related morbidity, mice were killed, and high leukemia infiltration was confirmed in bone marrow, spleen and PB by flow cytometry as previously described. ${ }^{8}$ 
RIP1 knockdown. Experiments were carried out $48 \mathrm{~h}$ after transient transfection (Amaxa Nucleofector, Lonza, Basel, Switzerland) with siRNA oligonucleotides (ON-TARGETplus Human RIP1 siRNA SMART pool and NON-TARGETING pool; Dharmacon, Lafayette, USA). REH cells with stable (shRNA) RIP1 knockdown were kindly provided by S Löder and Dr. Fulda, Ulm, Germany. ${ }^{29}$

Reagents, analysis of cell death signaling. Genentech (San Francisco, USA) kindly provided BV6, Pfizer (New York, USA) provided etanercept, Medac (Hamburg, Germany) provided E. coli asparaginase and the Pharmacy, University Medical Center Ulm provided dexamethasone and vincristine. N-BenzyloxycarbonylVal-Ala-Asp-fluoromethylketone (z.VAD.fmk) was obtained from Bachem (Heidelberg, Germany), Nec-1 from Biomol (Hamburg, Germany) and all other chemicals from Sigma-Aldrich. Western blot analysis was performed as previously described, ${ }^{42}$ using mouse anti-caspase-8 (\#ALX-804-242-C100; Alexis Life Sciences, New York, USA); mouse anti-caspase-2 (\#611022), rabbit anticaspase-9 (\#556585), mouse anti-RIP (\#551041), mouse anti-FADD (\#610400; BD Biosciences), rabbit anti-caspase-3 (\#9662), rabbit anti-NIK (\#4994), mouse anti-plkBa (\#9246), rabbit anti-lkBa (\#9242; Cell Signaling, Beverly, USA), rabbit anti-caspase-8 (\#1006-1), rabbit anti-clAP2 (\#ab32059; Epitomics, Burlingame, USA), goat anti-cIAP1 (\#AF8181; R\&D Systems, Wiesbaden, Germany); mouse anti-p100/p52 (\#05-361; Merck Millipore, Darmstadt, Germany), mouse anti-betaactin (\#A5441; Sigma-Aldrich, Steinheim, Germany); followed by horseradish peroxidase-conjugated goat anti-mouse (SC-2005), goat anti-rabbit (SC-2004) or donkey anti-goat (SC2020; Santa Cruz Biotechnology, Santa Cruz, USA) antibodies. For immunoprecipitation, cells were lysed in $10 \mathrm{mM}$ Tris (pH 8.0), $150 \mathrm{mM} \mathrm{NaCl}$ and $1 \%$ Nonidet P-40, supplemented with protease inhibitor (Roche, Grenzach, Germany), and incubated $\left(4^{\circ} \mathrm{C}\right)$ with mouse anti-caspase-8 (as above) and panmouse-IgG Dynabeads (\#11041; Invitrogen/Life Technologies, Darmstadt, Germany), washed, and analyzed by western blot. Cell death, cytochrome- $c$ release, activated caspase- 3 and mitochondrial membrane potential were assessed as previously described. ${ }^{7,9,10,43}$ TNF- $\alpha$ was measured in cellular supernatants by a bead-based immunoassay (Fluorokine MAP cytokine, R\&D Systems) and a BioPlex analyzer (Bio-Rad, Munich, Germany).

Statistical analysis. $I C_{50}$ were calculated based on three independent experiments performed in triplicates, different conditions obtained from three independent experiments performed in triplicates were compared by Mann-Whitney U-test (Prism 5.0, GraphPad, La Jolla, USA). Leukemia-free survival was analyzed by Kaplan-Meier and log-rank tests (SPSS 19.0, IBM, Munich, Germany), one recipient (VDA+BV6) moribund not due to leukemia was censored. $P$-values $<0.05$ were considered as significant.

\section{Conflict of Interest}

The authors declare no conflict of interest.

Acknowledgements. We thank Henning Walczak for helpful discussions, and Susann Baumgart, Manuel Hermann and Sandra Volk for excellent technical assistance. We thank Genentech, Roche and Medac for kindly providing research reagents. This work was supported by the Else Kröner-Fresenius-Foundation (2010_Kolleg24 to MS, FS, ET and SS), the Boehringer Ingelheim Fonds (to MS), International Graduate School in Molecular Medicine UIm (to SD), the German Research Foundation (SFB1074, B6 to LHM and K-MD; B1 and B2 to SS), the Helmholtz Association/Preclinical Comprehensive Cancer Center (K-MD) and the 'Förderkreis für Tumor- und Leukämiekranke Kinder Ulm'.

1. Pui CH, Evans WE. Treatment of acute lymphoblastic leukemia. N Engl J Med 2006; 354: 166-178.

2. Pui $\mathrm{CH}$, Carroll WL, Meshinchi S, Arceci RJ. Biology, risk stratification, and therapy of pediatric acute leukemias: an update. J Clin Oncol 2011; 29: 551-565.

3. Schrappe M, Reiter A, Ludwig WD, Harbott J, Zimmermann M, Hiddemann W et al. Improved outcome in childhood acute lymphoblastic leukemia despite reduced use of anthracyclines and cranial radiotherapy: results of trial ALL-BFM 90. German-AustrianSwiss ALL-BFM Study Group. Blood 2000; 95: 3310-3322.

4. Stanulla M, Cario G, Meissner B, Schrauder A, Moricke A, Riehm $\mathrm{H}$ et al. Integrating molecular information into treatment of childhood acute lymphoblastic leukemia-a perspective from the BFM Study Group. Blood Cells Mol Dis 2007; 39: 160-163.

5. Fulda S. Tumor resistance to apoptosis. Int J Cancer 2009; 124: 511-515.
6. Hanahan D, Weinberg RA. Hallmarks of cancer: the next generation. Cell 2011; 144: 646-674.

7. Queudeville M, Seyfried F, Eckhoff SM, Trentin L, Ulrich S, Schirmer M et al. Rapid engraftment of human ALL in NOD/SCID mice involves deficient apoptosis signaling. Cell Death Dis 2012; 3: e364.

8. Meyer LH, Eckhoff SM, Queudeville M, Kraus JM, Giordan M, Stursberg J et al. Early relapse in ALL is identified by time to leukemia in NOD/SCID mice and is characterized by a gene signature involving survival pathways. Cancer Cell 2011; 19: 206-217.

9. Meyer LH, Karawajew L, Schrappe M, Ludwig WD, Debatin KM, Stahnke K. Cytochrome c-related caspase-3 activation determines treatment response and relapse in childhood precursor B-cell ALL. Blood 2006; 107: 4524-4531.

10. Meyer LH, Queudeville M, Eckhoff SM, Creutzig U, Reinhardt D, Karawajew L et al. Intact apoptosis signaling in myeloid leukemia cells determines treatment outcome in childhood AML. Blood 2008; 111: 2899-2903.

11. Fulda S, Debatin KM. Extrinsic versus intrinsic apoptosis pathways in anticancer chemotherapy. Oncogene 2006; 25: 4798-4811.

12. Ashkenazi A. Targeting the extrinsic apoptosis pathway in cancer. Cytokine Growth Factor Rev 2008; 19: 325-331.

13. Brenner D, Mak TW. Mitochondrial cell death effectors. Curr Opin Cell Biol 2009; 21: 871-877.

14. Berthelet J, Dubrez L. Regulation of apoptosis by inhibitors of apoptosis (IAPs). Cells 2013; 2: $163-187$.

15. Hunter AM, LaCasse EC, Korneluk RG. The inhibitors of apoptosis (IAPs) as cancer targets. Apoptosis 2007; 12: 1543-1568.

16. Dubrez L, Berthelet J, Glorian V. IAP proteins as targets for drug development in oncology. Onco Targets Ther 2013; 9: 1285-1304.

17. Wuchter C, Richter S, Oltersdorf D, Karawajew L, Ludwig WD, Tamm I. Differences in the expression pattern of apoptosis-related molecules between childhood and adult de novo acute myeloid leukemia. Haematologica 2004; 89: 363-364.

18. Hundsdoerfer P, Dietrich I, Schmelz K, Eckert C, Henze G. XIAP expression is posttranscriptionally upregulated in childhood ALL and is associated with glucocorticoid response in T-cell ALL. Pediatr Blood Cancer 2010; 55: 260-266.

19. Mannhold R, Fulda S, Carosati E. IAP antagonists: promising candidates for cancer therapy. Drug Discov Today 2010; 15: 210-219.

20. Fulda S. Inhibitor of apoptosis (IAP) proteins in hematological malignancies: molecular mechanisms and therapeutic opportunities. Leukemia 2014; 28: 1414-1422.

21. Fulda $S$, Vucic D. Targeting IAP proteins for therapeutic intervention in cancer. Nat Rev Drug Discov 2012; 11: 109-124.

22. Vince JE, Wong WW, Khan N, Feltham R, Chau D, Ahmed AU et al. IAP antagonists target CIAP1 to induce TNFalpha-dependent apoptosis. Cell 2007; 131: 682-693.

23. Varfolomeev E, Blankenship JW, Wayson SM, Fedorova AV, Kayagaki N, Garg P et al. IAP antagonists induce autoubiquitination of c-IAPs, NF-kappaB activation, and TNFalphadependent apoptosis. Cell 2007; 131: 669-681.

24. Wang L, Du F, Wang X. TNF-alpha induces two distinct caspase-8 activation pathways. Cell 2008; 133: 693-703.

25. Probst BL, Liu L, Ramesh V, Li L, Sun H, Minna JD et al. Smac mimetics increase cancer cell response to chemotherapeutics in a TNF-alpha-dependent manner. Cell Death Differ 2010; 17: $1645-1654$

26. Gaither A, Porter D, Yao Y, Borawski J, Yang G, Donovan J et al. A Smac mimetic rescue screen reveals roles for inhibitor of apoptosis proteins in tumor necrosis factor-alpha signaling. Cancer Res 2007; 67: 11493-11498.

27. Greer RM, Peyton M, Larsen JE, Girard L, Xie Y, Gazdar AF et al. SMAC mimetic (JP1201) sensitizes non-small cell lung cancers to multiple chemotherapy agents in an IAP-dependent but TNF-alpha-independent manner. Cancer Res 2011; 71: 7640-7648.

28. Fakler M, Loeder S, Vogler M, Schneider K, Jeremias I, Debatin KM et al. Small molecule XIAP inhibitors cooperate with TRAIL to induce apoptosis in childhood acute leukemia cells and overcome Bcl-2-mediated resistance. Blood 2009; 113: 1710-1722.

29. Loder S, Fakler M, Schoeneberger H, Cristofanon S, Leibacher J, Vanlangenakker $\mathrm{N}$ et al. RIP1 is required for IAP inhibitor-mediated sensitization of childhood acute leukemia cells to chemotherapy-induced apoptosis. Leukemia 2012; 26: 1020-1029.

30. Weisberg E, Ray A, Barrett R, Nelson E, Christie AL, Porter D et al. Smac mimetics: implications for enhancement of targeted therapies in leukemia. Leukemia 2010; 24 : 2100-2109.

31. Scavullo C, Servida F, Lecis D, Onida F, Drago C, Ferrante L et al. Single-agent Smacmimetic compounds induce apoptosis in B chronic lymphocytic leukaemia (B-CLL). Leuk Res 2013; 37: 809-815.

32. Darding M, Meier P. IAPs: guardians of RIPK1. Cell Death Differ 2012; 19: 58-66.

33. Belz K, Schoeneberger $\mathrm{H}$, Wehner S, Weigert A, Bonig H, Klingebiel T et al. Smac mimetic and glucocorticoids synergize to induce apoptosis in childhood ALL by promoting ripoptosome assembly. Blood 2014; 124: 240-250.

34. Benetatos CA, Mitsuuchi Y, Burns JM, Neiman EM, Condon SM, Yu G et al. Birinapant (TL32711), a bivalent SMAC mimetic, targets TRAF2-associated clAPs, abrogates TNFinduced NF-kappaB activation, and is active in patient-derived xenograft models. Mol Cancer Ther 2014; 13: 867-879.

35. Houghton PJ, Kang MH, Reynolds CP, Morton CL, Kolb EA, Gorlick R et al. Initial testing (stage 1) of LCL161, a SMAC mimetic, by the Pediatric Preclinical Testing Program. Pediatr Blood Cancer 2012; 58: 636-639. 
36. Muller-Sienerth N, Dietz L, Holtz P, Kapp M, Grigoleit GU, Schmuck C et al. SMAC mimetic BV6 induces cell death in monocytes and maturation of monocyte-derived dendritic cells. PLoS One 2011; 6: e21556.

37. Schimmer AD, Estey EH, Borthakur G, Carter BZ, Schiller GJ, Tallman MS et al. Phase I/ll trial of AEG35156 X-linked inhibitor of apoptosis protein antisense oligonucleotide combined with idarubicin and cytarabine in patients with relapsed or primary refractory acute myeloid leukemia. J Clin Oncol 2009; 27: 4741-4746.

38. Schimmer AD, Herr W, Hanel M, Borthakur G, Frankel A, Horst HA et al. Addition of AEG35156 XIAP antisense oligonucleotide in reinduction chemotherapy does not improve remission rates in patients with primary refractory acute myeloid leukemia in a randomized phase II study. Clin Lymphoma Myeloma Leuk 2011; 11: 433-438.

39. Hasan MN, Queudeville M, Trentin L, Eckhoff SM, Bronzini I, Palmi C et al. Targeting of hyperactivated mTOR signaling in high-risk acute lymphoblastic leukemia in a pre-clinical model. Oncotarget 2015; 6: 1382-1395

40. Drexler HG. Guide to leukemia-lymphoma cell lines. 2nd Edition, Braunschweig, 2010.

41. Zenz T, Eichhorst B, Busch R, Denzel T, Habe S, Winkler D et al. TP53 mutation and survival in chronic lymphocytic leukemia. J Clin Oncol 2010; 28: 4473-4479.
42. Fulda S, Meyer E, Debatin KM. Metabolic inhibitors sensitize for CD95 (APO-1/Fas)-induced apoptosis by down-regulating Fas-associated death domain-like interleukin 1-converting enzyme inhibitory protein expression. Cancer Res 2000; 60: 3947-3956.

43. Vogler M, Durr K, Jovanovic M, Debatin KM, Fulda S. Regulation of TRAIL-induced apoptosis by XIAP in pancreatic carcinoma cells. Oncogene 2007; 26: 248-257.

(c) (i) Cell Death and Disease is an open-access journal published by Nature Publishing Group. This work is licensed under a Creative Commons Attribution 4.0 International License. The images or other third party material in this article are included in the article's Creative Commons license, unless indicated otherwise in the credit line; if the material is not included under the Creative Commons license, users will need to obtain permission from the license holder to reproduce the material. To view a copy of this license, visit http://creativecommons.org/licenses/by/4.0/

Supplementary Information accompanies this paper on Cell Death and Disease website (http://www.nature.com/cddis) 